\title{
Clinical analysis of the patients with osseous metastatic breast cancer
}

\author{
Hyung-Seok Park', Yong Jin Cho ${ }^{2}$ \\ ${ }^{1}$ Department of Orthopedic Surgery, Seoul Saint Mary's Hospital, Seoul, ${ }^{2}$ Department of Orthopedic Surgery, Chosun University College \\ of Medicine, Gwangju, Korea
}

Received December 4, 2020

Revised December 10, 2020

Accepted December 10, 2020

Corresponding author

Yong Jin Cho

Department of Orthopedic Surgery,

Chosun University Hospital, 365

Pilmun-daero, Dong-gu, Gwangju

61453, Korea

Tel: +82-62-220-3147

Fax: +82-62-226-3379

E-mail: choisidoru@chosun.ac.kr

ORCID:

https://orcid.org/0000-0002-6044-627X
The incidence of osseous metastatic breast cancer is on the rise. However, there are few systematic studies, particularly in patients with osseous metastatic breast cancer after orthopedic surgery. We performed a medical record review of 34 patients who underwent orthopedic surgery for osseous metastasis from breast cancer during 2012. 1-2015. 1. The average age was 48.5 (27-74). A total of 49 orthopedic surgeries were done. The femur was the most common site for orthopedic surgery for osseous metastasis (17 cases, 34.6\%). And the most common surgery was curettage, bone cementing and intramedullary nailing (23 cases, 46.9\%). Estrogen receptor positive was the most common case with 25 cases (73.5\%), triple negative was 3 cases (8.8\%). Mean survival time and median survival time were 15.5 months (4-36) and 10 months each after orthopedic surgery. Orthopedic surgery should be considered depending on the condition, after careful evaluation of the patients with osseous metastasis due to breast cancer.

Keywords: Incidence; Bone neoplasms; Orthopedics; Survival rate

\section{INTRODUCTION}

Cancer is the main cause of death worldwide. In 2012, about 14 million individuals were diagnosed with cancer and 8.2 million cancer-related deaths were reported [1]. The incidence of cancer in Korea, including breast cancer, has increased due to population aging and westernization of lifestyle in recent year [2]. According to the cancer registration report issued by the Ministry of Health and Welfare, breast cancer ranked as the third most common cancer in women before 1998, became the most common cancer in 2017, and accounted for about $20.3 \%$ of all cancer in Korean women in 2017 [2-4]. Moreover, the incidence of breast cancer has continued to rise in the last several decades [4].
Fortunately, due to recent advances in systemic therapy, the survival rate of breast cancer patients is increasing regardless of whether or not bone metastasis is present [5]. As a consequence, the prevalence of patients with a history of breast cancer is increasing as the rate of survival improves, making breast cancer in many aspects a chronic condition [6].

Recently, studied on the hormonal receptor mutation status for breast cancer have been actively conducted, leading to the development of the chemotherapy or targeted therapies. Although somewhat controversial, the metastasis pattern of breast cancer varies by hormone receptor status. It is known that metastasis of other organs including the brain is fast and the overall treatment effect is also poor in case of triple negative, namely estrogen receptor (ER) negative, pro- 
gesterone receptor (PR) negative, human epidermal growth factor receptor2 (HER2) negative. Long-term survival rates are relatively higher in patients with ER positive, but bone metastasis is reported to be more frequent [7].

Breast cancer frequently metastasizes to the bone, and around $70 \%$ of patients with recurrent breast cancer have bone metastasis, a common cause of morbidity and mortality [8]. So that, when breast cancer metastasis to other organs, bone metastasis is almost always present, and such bone related events such as pain, pathological fracture, and hypercalcemia are not rare clinical complications [0].

Despite the trend of bone metastasis in breast cancer, there are few research results related to the patients with osseous metastatic breast cancer. In this study, so we performed a retrospective study to evaluate the clinical features, hormone receptor status, orthopedic treatment and its results in patients of breast cancer with long bone metastasis.

\section{MATERIALS AND METHODS}

The study was based on a consecutive series of breast cancer patients operated for osseous metastasis during 2012. 1-2015. 1 at Bone and Soft tissue sarcoma Clinic at the Yonsei University College of Medicine, Seoul, Korea. The study was conducted in agreement with the guidelines and approved by the Ethics Committee of the Chosun University Hospital (CHOSUN 2020-06-001). The breast cancer was diagnosed based on histologic analysis of biopsy and open surgical specimen. Osseous metastasis and other organ metastasis were confirmed via positive bone scan. This included patients who were operated for osseous metastatic breast cancer irrespective of whether spine is involved. Indication for orthopedic operation of the limbs was complete or impending skeletal fractures. We excluded patients with other primary cancers, patients without orthopedic surgery, and patients who were dropped out during follow-up. Duration from diagnosis to final follow-up, age at diagnosis of primary cancer, outcome of primary cancer pathology, size, whether it is menopause at diagnosis, hormonal receptor mutation status of primary cancer, other organ metastasis sites, location of the osseous metastasis of the limbs, type of orthopedic surgery and the results were collected and analyzed.

\section{RESULTS}

Osseous metastasis occurred in 34 cases, all cases were female, and the average age was 48.5 years (27-74) (Table 1). 20 (58.8\%) underwent one surgery, 13 (38.2\%) underwent two surgeries, and one (2.9\%) underwent three surgeries (Fig. 1). A total of 49 operations were performed. The most common surgery for osseous metastasis was curettage with bone cementing and intramedullary nailing, performed in 23 cases (46.9\%), 12 cases for femur trochanter and shaft, 10 cases for humerus, and one case for tibia. There were 13 cases arthroplasty surgeries. There were 10, two, one cases of hip joint, shoulder joint and knee joint, respectively. There were five cases of curettage with plate fixation on the femur shaft. The curettage with bone cementing was performed in five cases in the pelvis. The excision was performed in three cases at the supraclavicular area. The most common surgical sites were femur in 17 cases (34.6\%), hip joint and humerus in 10 cases, and pelvis in five cases (Table 2). Complication related to surgery was not observed, and case of fixation failure before death was not found, either.

At the time of diagnosis of primary breast cancer, there were 21 cases (61.8\%) of lymph node metastasis and 18 cases (52.9\%) of menopause (Table 1). According to the pathological results of primary breast cancer surgery, the histological grade was I in 10 cases (29.4\%), II in 12 cases (35.3\%), and III in 12 cases (35.3\%). Lobular carcinoma was found in two patients (5.9\%), mucous adenocarcinoma in one patient (2.9\%), and invasive ductal carcinoma in 31 patients (91.2\%). The size of the primary lesion was less than $2 \mathrm{~cm}$ in 12 cases (35.3\%), 2 to $5 \mathrm{~cm}$ in 19 cases (55.9\%), and more than 5

Table 1. Baseline characteristics of 34 breast cancer patients

\begin{tabular}{lc}
\hline \multicolumn{1}{c}{ Variables } & Value \\
\hline Patients & $34(100 \%)$ \\
Female & $0(0 \%)$ \\
Male & $48.5(27-74)$ \\
Age & \\
Menopause at diagnosis & $18(52.9 \%)$ \\
Yes & $16(47.1 \%)$ \\
No & \\
Regional lymph node involvement & $21(61.8 \%)$ \\
Yes & $13(38.2 \%)$ \\
No
\end{tabular}



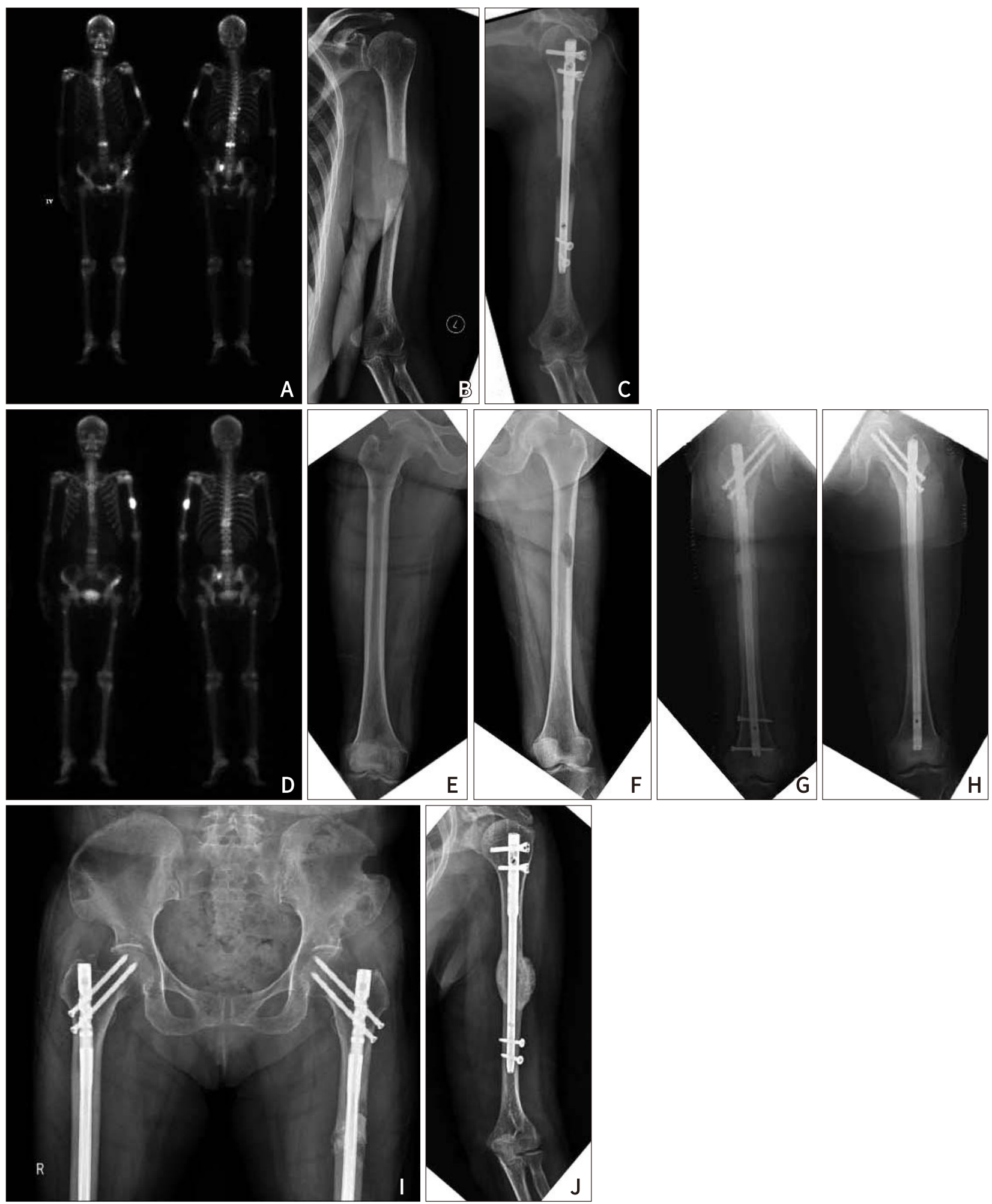

Fig. 1. A case of 63 years old female patient who underwent 3 surgeries. (A) A whole body bone scan shows increased uptake on left humerus, (B and C) a plain radiography of preoperative and postoperative of the left humerus, curettage with bone cementing and intramedullary nailing was done, (D) a whole body bone scan after 6 months from the surgery of the left humerus shows increased uptake on both femur shaft, (E, F and G, H) a plain radiography of preoperative and postoperative of the both femur, curettage with bone cementing and intramedullary nailing was done, (I and J) a plain radiography after 12 months from the femur surgery. She was able to walk independently with one cane support. 
Table 2. Orthopedic operation for osseous metastasis

\begin{tabular}{lr}
\multicolumn{1}{c}{ Variables } & Value \\
\hline A number of operation & 20 \\
1 operation & 13 \\
2 operations & 1 \\
3 operations & \\
Location & 3 \\
Supraclavicular area & 2 \\
Shoulder & 10 \\
Humerus & 5 \\
Pelvis & 10 \\
Hip & 17 \\
Femur trochanter and shaft & 1 \\
Knee & 1 \\
Tibia & \\
Operation & 13 \\
Arthroplasty & 23 \\
Curettage with bone cementing, IM nailing & 5 \\
Curettage with bone cementing, plate fixation & 5 \\
Curettage and cementing & 3 \\
Excision & \\
\hline
\end{tabular}

Table 3. Histopathologic characteristics

\begin{tabular}{lc}
\multicolumn{1}{c}{ Variables } & Value \\
\hline Histologic grade & $10(29.4 \%)$ \\
\hline II & $12(35.3 \%)$ \\
Pathology & $12(35.3 \%)$ \\
Infiltrating ductal carcinoma & $31(91.2 \%)$ \\
Lobular carcinoma & $2(5.9 \%)$ \\
Mucinous adenocarcinoma & $1(2.9 \%)$ \\
Size (cm) & $12(35.3 \%)$ \\
$<2$ & $19(55.9 \%)$ \\
$2-5$ & $3(8.8 \%)$ \\
$5<$ & \\
Other organ metastasis at diagnosis & $13(38.2 \%)$ \\
Yes & $21(61.8 \%)$ \\
No &
\end{tabular}

$\mathrm{cm}$ in three cases (8.8\%). At the time of diagnosis, 13 cases (38.2\%) metastasized to other organs and 21 cases (61.8\%) metastasized to other organs during the follow-up period (Table 3). There were 14 liver metastasis (41.2\%), 16 lung metastasis (47.1\%), and five intracranial metastasis (14.7\%) excluding osseous metastasis at the last follow-up.

Hormonal receptor mutation status of primary carcinoma
Table 4. The results of tissue microarrays

\begin{tabular}{|c|c|}
\hline Variables & Value \\
\hline \multicolumn{2}{|l|}{ ER receptor } \\
\hline Positive & $25(73.5 \%)$ \\
\hline Negative & $9(26.5 \%)$ \\
\hline \multicolumn{2}{|l|}{ HER2 receptor } \\
\hline Positive & $10(29.4 \%)$ \\
\hline Negative & $24(70.6 \%)$ \\
\hline \multicolumn{2}{|l|}{ PR receptor } \\
\hline Positive & $18(52.9 \%)$ \\
\hline Negative & $16(47.1 \%)$ \\
\hline Triple negative & $3(8.8 \%)$ \\
\hline ER negative and HER2 positive & $3(8.8 \%)$ \\
\hline
\end{tabular}

ER: estrogen, HER2: human epidermal growth factor 2, PR: progesterone.

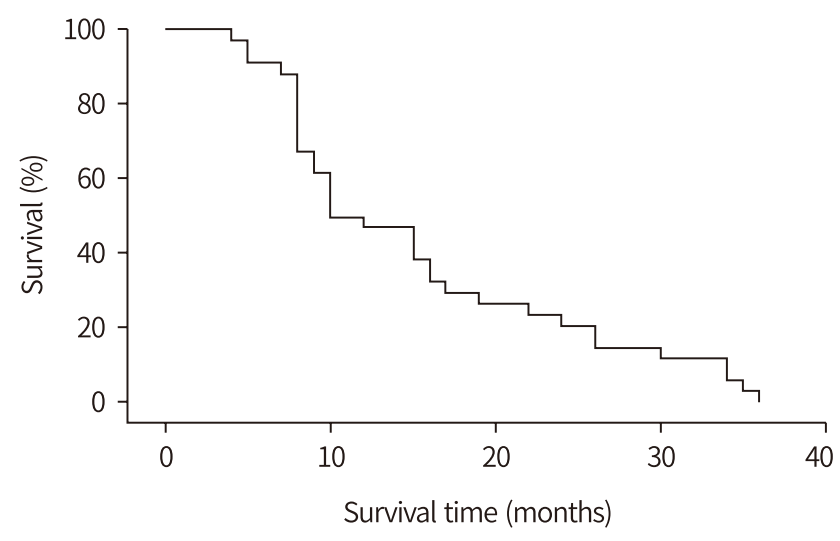

Fig. 2. Kaplan-Meier survival analysis of breast cancer patients with osseous metastasis after surgery.

is 25 cases $(73.5 \%)$ of ER positive, 10 cases of HER2 positive (29.4\%), 3 cases of Triple negative (8.8\%), ER was negative while HER2 was positive in 3 cases (8.8\%). PR positive was 18 cases $(52.9 \%)$ (Table 4 ). The average survival period of triple negative patients was 13 months (8-19). It took an average of 30 months (0-140) for osseous metastasis to be detected from the time of primary carcinoma diagnosis. Osseous metastasis was found at an average of 29 months in triple negative cases, while at 57 months in other cases. There was, however, no statistical significance due to the short follow-up period and few cases.

At the last follow-op, all patients died, and mean survival time was 15.5 months (4-36) and median survival time was 10 months after orthopedic surgery. The 1-year postoperative survival rate was $47.1 \%$, and the 2-year postoperative survival rate was $20.6 \%$ (Fig. 2). 


\section{DISCUSSION}

The numbers of newly diagnosed cancer cases and cancer-related deaths in 2017, in the Korea were 232,255 and 78,863 , respectively. Although overall cancer incidence and mortality have declined since 2011 and 2002, respectively, some cancers, such as breast and prostate cancer, demonstrate increasing trends with respect to both incidence and mortality [4]. The frequency of osseous metastasis is $5 \%$ at the time of primary diagnosis $[10,11]$, however, osseous metastasis are found in one third of patients with recurrent disease [12].

According to Kelly et al. [13], 257 patients were enrolled in their study for metastatic disease of the long bones. Of the 257 patients, femoral metastasis was the highest as 182 cases. The revision rate for the operative intervention was $3 \%$, however, in-hospital mortality was $14 \%$. They insisted that the mortality rate does seem high, but the procedures were undertaken as palliative interventions and as part of a multipart of the patient's final care. They assumed that overall fixation methods appeared durable, so that, the high mortality rate may reflect delay in seeking orthopedic opinion.

Recently, there was a study to identify prognostic variables associated with survival in a large cohort of breast cancer patients after surgical treatment of osseous metastasis [5]. The study identified 301 individual patients who underwent 355 surgical procedures. The median age at the first surgical procedure was 61 (52-70) years. Approximately half of the patients had multiple skeletal metastases at surgery and most patients presented with complete pathological fractures. The femur was the most common site of surgery, accounting for 190 procedures (54\%). The cumulative 1-, 2-, and 5-year survival rates after surgery were $45 \%, 27 \%$, and $8 \%$, respectively. At the end of the study, $90 \%$ of the patients had died (median follow-up time 1 year). The overall complication rate was $25 \%$. The re-operation rate was $14 \%$. The most common reason for re-operation was implant failure. They revealed an increased risk of death after surgery for over 60 years of age and low hemoglobin levels. According to the clinical analysis of patients with skeletal metastasis of lung cancer, also, patients who survived longer were less than 60 years old [14]. The older age was not seen by others $[15,16$, however, low hemoglobin has been shown in patients with other primary tumors $[15,17]$.
As a result of studies on the clinical characteristics of breast cancer [7], it was known that other organ metastasis are found on average 30 months after breast cancer diagnosis, and within 5 years after diagnosis of primary cancer, $75.8 \%$ of other organ metastases are found. In the correlation analysis of hormonal receptor mutation status for breast cancer metastasis pattern, it was reported that median overall survival of patients with both ER and HER2 negative is significantly shorter than that of patients with $E R$ negative and HER2 positive or ER positive, 27 and 52, 76 or 79 , respectively. In recent study while the incidence of osseous metastasis was significantly higher in HR positive and HER2 positive than in patients with different subtypes [18], patients with a triple-negative subtype were, most likely to develop visceral metastases rather than osseous metastasis [18,19].

Some studies suggested that female breast cancer survival may be associated with different metastasis patterns [20-22]. Errani et al. reported that breast cancer patients with lungs, the first site of distant metastasis, had the highest survival rate (58.5 months) compared to bone (44.4 months), liver (36.7 months), or brain (7.35 months) with first metastasis confirmed [21]. The 5-year survival rate was, however, significantly increased in patients with bone metastases compared to other metastases in other studies [21]. Cleeland et al. have shown that patients with bone metastasis have the best prognosis and brain metastasis is the most aggressive subgroup in their study [19]. The clinical treatment of osseous metastasis from breast cancer is essentially aimed at palliating pain, maintaining function and preventing skeletal-related events [23-25]. Systemic therapies that block osteoclast activity, including bisphosphonates (zoledronic acid) and receptor activator for nuclear factor ${ }^{-} \kappa \beta$ ligand (RANKL) inhibitors (denosumab), can reduce the incidence of SREs [26,27]. The recent development of remarkable chemotherapy/immunotherapy and supportive therapies has caused the survival time in patients with breast cancer to dramatically increase, and interest in skeletal metastasis of breast cancer has increased [28]. In other words, breast cancer patients will survive longer and inhibited SREs by the use of bisphosphonates and RANKL inhibitors, advanced chemotherapy including immunotherapy.

The number of long bone metastasis is increasing in breast cancer patients, and mutation status of hormone 
receptor plays a very important role in prognosis. It is often necessary, also, to perform surgery under general anesthesia multiple times on the other part of the same patient. Orthopedic surgery should be considered depending on the condition, after careful evaluation of the patients with osseous metastasis due to breast cancer.

\section{ACKNOWLEDGMENTS}

This study was supported by research fund from Chosun University Hospital, 2018.

\section{CONFLICT OF INTEREST}

No potential conflict of interest relevant to this article was reported.

\section{REFERENCES}

1. Ferlay J, Colombet M, Soerjomataram I, Dyba T, Randi G, Bettio $\mathrm{M}$, et al. Cancer incidence and mortality patterns in Europe: Estimates for 40 countries and 25 major cancers in 2018. Eur J Cancer 2018;103:356-87.

2. Ministry of Health and Welfare. Annual report of cancer registry programme in the republic of Korea, January 1 to December 31, 2016. Sejong: Ministry of Health and Welfare; 2019.

3. Ahn SH; Korean Breast Cancer Society. Clinical characteristics of breast cancer patients in Korea in 2000. Arch Surg 2004;139:27-30; discussion 31.

4. Hong S, Won YJ, Park YR, Jung KW, Kong HJ, Lee ES; Community of Population-Based Regional Cancer Registries. Cancer Statistics in Korea: incidence, mortality, survival, and prevalence in 2017. Cancer Res Treat 2020;52:335-50.

5. Weiss RJ, Tullberg E, Forsberg JA, Bauer HC, Wedin R. Skeletal metastases in 301 breast cancer patients: patient survival and complications after surgery. Breast 2014;23:286-90.

6. Ju DG, Yurter A, Gokaslan ZL, Sciubba DM. Diagnosis and surgical management of breast cancer metastatic to the spine. World J Clin Oncol 2014;5:263-71.

7. Savci-Heijink CD, Halfwerk H, Hooijer GK, Horlings HM, Wesseling J, van de Vijver MJ. Retrospective analysis of metastatic behaviour of breast cancer subtypes. Breast Cancer Res Treat 2015;150:547-57.

8. Arican A, Bozkurt T, Bozcuk H, Demirkan B, Buyukberber S, Alkis $\mathrm{N}$, et al. A cross-sectional survey of the diagnosis and management of bone metastasis in breast cancer patients in
Turkey. Support Care Cancer 2014;22:2629-34.

9. Coleman RE. Skeletal complications of malignancy. Cancer 1997;80(8 Suppl):1588-94.

10. Jemal A, Bray F, Center MM, Ferlay J, Ward E, Forman D. Global cancer statistics. CA Cancer J Clin 2011;61:69-90.

11. Jensen AØ, Jacobsen JB, Nørgaard M, Yong M, Fryzek JP, Sørensen HT. Incidence of bone metastases and skeletal-related events in breast cancer patients: a population-based cohort study in Denmark. BMC Cancer 2011;11:29.

12. Kunkler IH, Merrick MV. The value of non-staging skeletal scintigraphy in breast cancer. Clin Radiol 1986;37:561-2.

13. Kelly M, Lee M, Clarkson P, O'Brien PJ. Metastatic disease of the long bones: a review of the health care burden in a major trauma centre. Can J Surg 2012;55:95-8.

14. Nathan SS, Healey JH, Mellano D, Hoang B, Lewis I, Morris $\mathrm{CD}$, et al. Survival in patients operated on for pathologic fracture: implications for end-of-life orthopedic care. J Clin Oncol 2005;23:6072-82.

15. Sciubba DM, Gokaslan ZL, Suk I, Suki D, Maldaun MV, McCutcheon IE, et al. Positive and negative prognostic variables for patients undergoing spine surgery for metastatic breast disease. Eur Spine J 2007;16:1659-67.

16. Weiss RJ, Wedin R. Surgery for skeletal metastases in lung cancer. Acta Orthop 2011;82:96-101.

17. Wu Q, Li J, Zhu S, Wu J, Chen C, Liu Q, et al. Breast cancer subtypes predict the preferential site of distant metastases: a SEER based study. Oncotarget 2017;8:27990-96.

18. Pulido C, Vendrell I, Ferreira AR, Casimiro S, Mansinho A, Alho I, et al. Bone metastasis risk factors in breast cancer. Ecancermedicalscience 2017;11:715.

19. Cleeland C, von Moos R, Walker MS, Wang Y, Gao J, ChavezMacGregor M, et al. Burden of symptoms associated with development of metastatic bone disease in patients with breast cancer. Support Care Cancer 2016;24:3557-65.

20. Costa L, Badia X, Chow E, Lipton A, Wardley A. Impact of skeletal complications on patients' quality of life, mobility, and functional independence. Support Care Cancer 2008;16:879-89.

21. Errani C, Mavrogenis AF, Cevolani L, Spinelli S, Piccioli A, Maccauro G, et al. Treatment for long bone metastases based on a systematic literature review. Eur J Orthop Surg Traumatol 2017;27:205-11.

22. Araki N, Chuman H, Matsunobu T, Tanaka K, Katagiri H, Kunisada T, et al. Factors associated with the decision of operative procedure for proximal femoral bone metastasis: questionnaire survey to institutions participating the Bone and Soft Tissue Tumor Study Group of the Japan Clinical Oncology Group. J Orthop Sci 2017;22:938-45.

23. Tsuda Y, Yasunaga H, Horiguchi H, Fushimi K, Kawano H, Tanaka S. Complications and postoperative mortality rate 
after surgery for pathological femur fracture related to bone metastasis: analysis of a nationwide database. Ann Surg Oncol 2016;23:801-10.

24. Janssen SJ, Teunis T, Hornicek FJ, van Dijk CN, Bramer JA, Schwab JH. Outcome after fixation of metastatic proximal femoral fractures: a systematic review of 40 studies. J Surg Oncol 2016;114:507-19.

25. Plunkett TA, Smith P, Rubens RD. Risk of complications from bone metastases in breast cancer. implications for management. Eur J Cancer 2000;36:476-82.

26. Galvano A, Scaturro D, Badalamenti G, Incorvaia L, Rizzo S, Castellana L, et al. Denosumab for bone health in prostate and breast cancer patients receiving endocrine therapy? A systematic review and a meta-analysis of randomized trials. J Bone Oncol 2019;18:100252.

27. Henry DH, Costa L, Goldwasser F, Hirsh V, Hungria V, Prausova J, et al. Randomized, double-blind study of denosumab versus zoledronic acid in the treatment of bone metastases in patients with advanced cancer (excluding breast and prostate cancer) or multiple myeloma. J Clin Oncol 2011;29: 1125-32.

28. Stopeck AT, Fizazi K, Body JJ, Brown JE, Carducci M, Diel I, et al. Safety of long-term denosumab therapy: results from the open label extension phase of two phase 3 studies in patients with metastatic breast and prostate cancer. Support Care Cancer 2016:24:447-55. 